Monatsschrift für Geburtshülfe u. Gynäkologie 1908;28:717

\title{
George M. Edebohls †
}

Früh im Herbst dieses Jahres ist Edebohls von seinem langen Leiden erlöst worden. Schon im Frühjahr, als ich in New York auch ihn aufsuchen wollte, wurde mir mitgeteilt, dass er seit Monaten Nie-mand empfange, dass er einem unabwendbaren Siechtum erliege. So ist er denn erlöst worden!

Der Name Edebohls ist uns Gynäkologen seit langen Jahren wohl vertraut. Wir wissen, dass er an dem Postgraduate Hospital einer der erfolgreichsten Vertreter unseres Faches war, dass er als ein her-vorragender Operateur im Vordergrund stand. Ich selbst habe das Glück gehabt, ihn aus mehrfacher Begegnung persönlich schätzen zu lernen, er war ein liebenswürdiger Gentleman, voll tiefen wissenschaft-lichen Ernstes und Strebens.

Weitesten Kreisen wurde sein Name geläuñg, als er auf Grund sehr ernster Studien und Experimente seine Vorschläge zu chirurgischer Behandlung der Brig $\lambda$ tschen Nierenerkrankung veröffentlichte. Im be-sonderen hat die Dekortikation der Nieren bei Eklampsie seinen Namen gerade auch ausssrhalb unserer Fachgenossen bekannt gemacht. Die Akten sind über den Wert dieser Operation noch nicht geschlossen; dass sie aber der weiteren Pruning in geeigneten Fallen wert ist, darüber besteht kein Zweifel.

Edebohls, dessen Aussehen ein langes, erfolgreiches Leben prognostizieren liess, ist einem tückischen Siechtum erlegen. Um ihn trauern mit seiner engern Familie die Lehrer und besonders die Schüler des Post graduate Hospital in New York, denen er einer der ältesten und energischsten Förderer war. Aber auch weit darüber hinaus wird sein Andenken in Ehren bleiben: alle, welche mit ihm in persönliche Beziehungen gekommen, werden dem liebenswürdigen Mann und anregenden Kollegen in treuem Gedenken nachtrauern! A. Martin. 